Abós, P. \& Domingo, V. (2013). La diversidad territorial de España ante el reto de la atención a la diversidad: diversidad de políticas públicas. Revista Electrónica Interuniversitaria de Formación del Profesorado, 16 (2), 55-75.

\title{
La diversidad territorial de España ante el reto de la atención a la diversidad: diversidad de políticas públicas
}

\author{
Pilar Abós Olivares, Virginia Domingo Cebrián \\ Facultad de Ciencias Sociales y Humanas, Universidad Zaragoza
}

\section{Resumen}

Las diferencias humanas proceden tanto de la desigualdad que produce la propia naturaleza como del origen y situación social y cultural y requieren un tratamiento educativo diferenciado e integrador. El estudio analiza las políticas educativas de once Comunidades Autónomas Españolas en relación al concepto de atención a la diversidad de acuerdo a los principios de la LOE, a través del análisis de la normativa vigente y de la descripción de los planes y programas existentes. Las diferencias encontradas se refieren fundamentalmente a la existencia o no de normativa posterior a la LOE y al desarrollo curricular autonómico, a la distinción o no entre programas de atención a la diversidad y de educación compensatoria, tanto en cuanto al alumnado al que van dirigidos, como en cuanto a las medidas adoptadas. En sentido contrario los puntos de acuerdo se centran en una concepción amplia de la atención a la diversidad bajo la que se engloban diferentes planes, proyectos, programas y medidas, todos ellos relacionados con la calidad del sistema educativo y el logro del éxito por parte del alumnado.

\section{Palabras clave}

Atención a la diversidad; compensación educativa; éxito escolar; apoyo y refuerzo escolar. 


\title{
Spain's territorial diversity facing the challenge of attention to diversity: a survey on the differences in the implementation of public policies
}

\begin{abstract}
Social origin and cultural diversity make up the differences between human beings. In this particular context, educational treatment towards those differences has evolved, trying to diminish them in terms of access to education. This research intends to analyze the educational policies of eleven autonomous regions of Spain, focusing on attention to diversity with regards to what LOE implementation means. To that purpose this research has made, on the one hand, a deep analysis of the differences of the educational laws and, on the other hand, we have tried to describe and analyze the specific educational programs that take place in the autonomous regions. We have found that the main differences come from the various ways in which LOE has been implemented, the degree of curricular development in each region and the similarities and dissimilarities in attention to diversity and compensatory education; and the importance of educational guidance in supporting policies for students. On the contrary, similarities stem from a wide conception of attention to diversity (different syllabuses, projects, programs and educational measures), but with a common goal: the improvement of the quality of the educational system and the achievement of success on behalf of the students.
\end{abstract}

\section{Key words}

Attention to diversity, compensatory education, school success, school support and reinforcement.

\section{Introducción}

Las diferencias humanas proceden, por un lado de la desigualdad que produce la propia naturaleza y por otro del estatuto social, que puede aumentarlas. La maduración del ser humano, como conjunto de procesos de crecimiento orgánico que conducen a la madurez y que se producen de modo ininterrumpido es fruto de la herencia y del aprendizaje, por lo que la progresiva adaptación del individuo al medio depende tanto de los procesos de crecimiento orgánico como del influjo del medio en el que se desenvuelve. Así, el proceso madurativo precisa de un proceso de estimulación que procede del exterior y posibilita el aprendizaje; el desarrollo funcional del sujeto se hace cada vez más complejo a medida que el medio gana en complejidad. Unido a todo ello está el hecho de que cada sujeto con su dotación genética, su medio y su experiencia es único, lo que explica las diferencias individuales.

En este contexto el tratamiento educativo dado a estas diferencias ha ido evolucionando a medida que la ciencia y la defensa de los derechos humanos han posibilitado una concepción diferente sobre las posibilidades de desarrollo y aprendizaje de sujetos con características, capacidades y posibilidades diversas. Desde el infanticidio, el rechazo, la repulsión, la ignorancia, la institucionalización.....hasta la integración y la compensación, la inclusión y la atención a la diversidad, se han escrito muchas páginas de la historia de la educación de los sujetos con condiciones personales o sociales "diferentes", páginas que nos conducen hasta la realidad actual en la que no podemos ser ajenos a la cultura de la diversidad. Ésta es lo más genuino y natural del ser humano y aceptarla supone concebirla 
como elemento de progreso y de riqueza en la construcción de una nueva escuela y una nueva sociedad (López Melero, 1997). Desde un punto de vista formal el principio de atención a la diversidad está basado en la obligación de los Estados y sus sistemas educativos de garantizar a todos el derecho a la educación (Dieterlen, 2001; Gordon, 2001), reconociendo la diversidad de sus necesidades, combatiendo las desigualdades y adoptando un modelo educativo abierto y flexible que permita el acceso y la permanencia escolar de todo el alumnado, sin excepción, así como resultados escolares aceptables (Organización de las Naciones Unidas para la Educación, la Ciencia y la Cultura, UNESCO, 1994).

Esta cultura de la diversidad debe estar presente en todas aquellas políticas educativas que defiendan una educación de calidad para todos y que tengan en cuenta sus necesidades y posibilidades, así como en las políticas de formación del profesorado, ya que resulta evidente la presencia de alumnos con diversas potencialidades y necesidades en nuestras aulas (LLorent, V y López, 2012) ; en este marco se ubica el objetivo del trabajo: describir, analizar y comparar algunas de las políticas educativas de atención a la diversidad existentes en las Comunidades Autónomas que poseen como única lengua oficial el castellano (Andalucía, Aragón, Asturias, Canarias, Cantabria, Castilla-La Mancha, CastillaLeón, Extremadura, La Rioja, Madrid y Murcia).

\section{Planteamiento del tema}

El traspaso de las competencias en materia educativa y, en consecuencia, el traspaso efectivo de los servicios educativos a las Comunidades Autónomas a partir de la Constitución de 1978 se rige, entre otros por los principios de unidad y de autonomía que implican la existencia de un estado único que garantiza la igualdad substancial de derechos y deberes de los ciudadanos y la homogeneidad básica del sistema educativo, así como la capacidad de cada Comunidad Autónoma para poder establecer políticas propias en temas educativos. En este sentido, y en relación con el tema que nos ocupa, el Estado debe garantizar que todo el alumnado pueda aprender y desarrollarse a partir de sus capacidades, pero serán los diferentes territorios los que diseñen y pongan en práctica políticas y programas educativos al servicio del citado objetivo. De este modo el marco normativo de la Ley Orgánica $2 / 2006$, de 3 de mayo, referente inmediato para todas las actuaciones de las Comunidades Autónomas aborda la intervención sobre una realidad socioeducativa evidente: la diversidad del alumnado, obligando a dar una respuesta institucional partiendo de que la educación es un servicio público que debe ser asequible a todos, sin distinción ninguna de condiciones personales y/o sociales. "La atención a la diversidad se establece como principio fundamental que debe regir toda la enseñanza básica, con el objetivo de proporcionar a todo el alumnado una educación adecuada a sus características y necesidades (p.17162) (...) La atención a la diversidad es una necesidad que abarca a todas las etapas educativas y a todos los alumnos. Es decir, se trata de contemplar la diversidad de las alumnas y alumnos como principio y no como una medida que corresponde a las necesidades de unos pocos" (p.17163). Al abrigo de este mandato el Estado de las Autonomías abre la oportunidad de rediseñar la respuesta y precisar medidas e instrumentos para el logro de los objetivos de una educación en igualdad de oportunidades, de modo que los poderes públicos puedan garantizar la prestación del servicio educativo con regularidad y adaptándose a los cambios sociales y a las necesidades del alumnado.

El enfoque dado al trabajo parte de la consideración que se hace del tema en la LOE (2006) y que supone que, partiendo del principio básico de atención a la diversidad, pueden y deben existir actuaciones de apoyo y/o compensación de carácter general o específico, que garanticen la equidad ( $p .17163$ ) y que dan lugar a dos grandes tipos de actuaciones: 
- Aquellas dirigidas al alumnado con necesidad específica de apoyo educativo entre los que incluimos a los alumnos que presentan necesidades educativas especiales, dificultades específicas de aprendizaje, altas capacidades intelectuales, incorporación tardía al sistema educativo o condiciones personales o de historia escolar (arts.71-77).

- Aquellas de carácter compensatorio dirigidas a personas, grupos y ámbitos territoriales que se encuentren en situaciones desfavorables y que sirvan para reforzar la acción del sistema educativo de forma que se eviten desigualdades derivadas de factores sociales, económicos, culturales, geográficos, étnicos o de otra índole (arts. 80-83).

Por este motivo el tema objeto de análisis han sido las políticas de atención a la diversidad y/o de compensación educativa desarrolladas por las diferentes CCAA que poniendo el énfasis en el reconocimiento de la diferencia como elemento integrante de cualquier proceso de enseñanza-aprendizaje, van dirigidas al logro del éxito educativo y a la mejora de los centros en definitiva al logro de una educación de calidad. Aunque en un principio la idea fue realizar el trabajo en las 17 Comunidades Autónomas, las dificultades para la comprensión de algunas fuentes y/o documentos que se encontraban escritos en las otras lenguas cooficiales, nos inclinó a centrarnos en las once comunidades cuya única lengua oficial es el castellano, al mismo tiempo que en la etapa de educación primaria.

De este modo el estudio pretende visibilizar el diseño de aquellas políticas, programas y actuaciones que se están desarrollando en algunas de las Comunidades Autónomas del Estado Español, dirigidas fundamental, aunque no exclusivamente, al alumnado de educación primaria y que, buscando el éxito de todo su alumnado, articulan respuestas educativas flexibles para dar respuesta a situaciones heterogéneas. Y todo ello a través de:

- La identificación de la normativa autonómica en relación a la atención a la diversidad y/o educación compensatoria

- La del planteamiento básico de las CCAA estudiadas respecto a la política de atención a la diversidad y/o educación compensatoria.

- El inventario de programas y planes de carácter autonómico en relación con la atención a la diversidad y/o educación compensatoria.

- La ubicación de los programas resultantes de convenios entre el Ministerio de Educación y las CCAA (ej. PROA) en el marco de los planes autonómicos.

\section{Normativa Autonómica}

\section{Descripción ordenada por orden cronológico de asunción de competencias}

En este apartado se detallan aquellos textos legales de carácter autonómico relacionados con la atención a la diversidad y/o la educación compensatoria. En aquellas CCAA en las que existan normas anteriores y posteriores a la LOE (2006), sólo haremos referencia a las segundas, aunque haremos referencia a aquellas otras que hayan servido de punto de partida de la normativa autonómica y que tengan un especial interés en relación a la temática abordada.

\section{Andalucía}

- Ley 9/1999, de 18 de noviembre, de Solidaridad en la Educación que abarca medidas y actuaciones de compensación de situaciones de desigualdad

- Decreto 167/2003, de 17 de junio por el que se establece la ordenación de la atención educativa a los alumnos y alumnas con necesidades educativas especiales asociadas a condiciones sociales desfavorecidas en el que se determinan un conjunto de medidas 
dirigidas al alumnado que se encuentra en situación de desventaja para el acceso, permanencia y promoción en el sistema educativo (entornos urbanos con problemática socioeducativa, determinadas zonas rurales, inmigrantes, comunidad gitana, hijos de temporeros o trabajadores itinerantes, imposibilidad de asistencia a los centros por razones judiciales o de enfermedad).

- Decreto 230/2007, de 31 de julio, por el que se establece la ordenación y las enseñanzas correspondientes a la educación primaria en Andalucía en el que se señala que la atención a la diversidad exige diferentes medidas curriculares y organizativas tanto ordinarias como específicas.

- Ley $17 / 2007$, de 10 de diciembre, de Educación de Andalucía, que reconoce el respeto a la diversidad del alumnado como principio básico del sistema educativo andaluz.

- Orden de 25 de julio de 2008 , por la que se regula la atención a la diversidad que cursa la educación básica en los centros docentes públicos de Andalucía, en la que se indica como principio la educación común y la atención a la diversidad poniendo énfasis en el desarrollo de competencias básicas, la detección y tratamiento precoz de las dificultades de aprendizaje, la tutoría y la orientación.

Aragón

- Decreto de 19 de diciembre de 2000 , de atención al alumnado con necesidades educativas especiales en el que incluye tanto alumnado que se encuentra en situaciones personales sociales o culturales desfavorecidas o que manifiesta dificultades graves de adaptación escolar (incluye niños hospitalizados o convalecientes), como al que presenta condiciones personales de discapacidad física, psíquica o sensorial o como consecuencia de una sobredotación intelectual.

- Orden de 9 de mayo de 2007 , por la que se aprueba el currículo de la educación primaria, que determina que las características del alumnado de esta etapa requieren una adecuada atención a la diversidad que permita garantizar una educación de calidad para todo el alumnado.

\section{Asturias}

- Decreto 56/2007, de 24 de mayo, que regula la ordenación y establece el currículo de Educación Primaria en el Principado de Asturias y plantea un modelo educativo que potencie la igualdad de oportunidades y el incremento de los niveles de calidad para todo el alumnado regido por principios como los de inclusión, normalidad, flexibilidad y contextualización.

\section{Canarias}

- Orden de 19 de mayo de 1995, por la que se establecen las acciones de carácter específico de compensación educativa en la Comunidad Autónoma de Canarias actuando sobre situaciones desfavorecidas y desfavorecedoras.

- Decreto 126/2007, de 24 de mayo, por el que se establece la ordenación y el currículo de la Educación Primaria y que señala la atención a la diversidad como principio básico de intervención que garantice una educación sólida para todo el alumnado persiguiendo su éxito personal.

- Orden de 7 de junio de 2010, por la que se regulan las condiciones que han de regir los planes, programas y proyectos de intervención dirigidos a mejorar la calidad y 
equidad en educación, de aplicación en los centros docentes no universitarios sostenidos con fondos públicos de la Comunidad Autónoma de Canarias y que tendrán como objetivos mejorar los resultados educativos, favorecer la cohesión social y reducir el abandono escolar, uniendo calidad con equidad.

- Decreto 104/2010, de 29 de julio, por el que se regula la atención a la diversidad del alumnado en el ámbito de la enseñanza no universitaria de Canarias y se reconoce y acepta la diversidad de necesidades y el necesario ajuste de respuestas.

- Orden de 13 de diciembre de 2010, por la que se regula la atención al alumnado con necesidades específicas de apoyo educativo en la Comunidad Autónoma de Canarias y se incluyen diferentes tipos de medidas: ordinarias, extraordinarias y excepcionales.

- Resolución de 9 de febrero de 2011, de la Dirección General de Ordenación, Innovación y Promoción educativa, por la que se dictan instrucciones sobre los procedimientos y los plazos para la atención educativa al alumnado con necesidades específicas de apoyo educativo en los centros escolares de la Comunidad Autónoma de Canarias, dando especial importancia a la detección temprana y a los programas preventivos de refuerzo.

\section{Cantabria}

- Orden de de 17 de junio de 2004, por la que se establecen las bases y se convoca a los Centros Educativos Públicos de Cantabria para la realización de actuaciones de Compensación Educativa, propiciando el desarrollo de programas de intervención global a favor de colectivos sociales y culturales desfavorecidos.

- Decreto 98/2005, de 18 de agosto, de ordenación de la atención a la diversidad en las enseñanzas escolares y la educación preescolar en Cantabria y que considera la diversidad como elemento enriquecedor e incluye al alumnado con necesidades derivadas de factores personales o sociales relacionados con situaciones de desventaja socioeconómica y sociocultural, con condiciones que dificultan la asistencia continuada al centro educativo, con sobredotación intelectual, con desconocimiento de la lengua y la cultura españolas, con discapacidad física, psíquica o sensorial, con trastornos graves de la personalidad, de la conducta o del desarrollo, o de graves retrasos o trastornos de la comunicación y del lenguaje.

- Decreto 56/2007, de 10 de mayo, por el que se establece el currículo de la Educación Primaria en la Comunidad Autónoma de Cantabria, buscando una educación de calidad para todo el alumnado desde una perspectiva inclusiva.

- Ley de Cantabria 6/2008, de 26 de diciembre, de Educación de Cantabria que considera la atención a la diversidad como una actuación de mejora del sistema educativo y diferencia entre la atención al alumnado con necesidad específica de apoyo educativo y las actuaciones de compensación de desigualdades en educación.

- Orden EDU/34/2009, de 6 de abril, por la que se regula el Plan de Refuerzo Educativo Complementario en el Sistema Educativo de Cantabria, orientado a dar respuesta al principio de equidad y justicia social, dirigiéndose a un alumnado que presenta unas características individuales y sociales que condicionan negativamente su éxito escolar y que, al mismo tiempo, podrían mejorar 
notablemente con un acompañamiento educativo especifico, ofrecido en el ámbito escolar.

\section{Castilla la Mancha}

- Decreto 138/2002, de 8 de octubre, por el que se ordena la respuesta educativa a la diversidad del alumnado en la Comunidad Autónoma de Castilla-La Mancha, entendiendo la atención a la diversidad como toda actuación dirigida a dar respuesta a las diferentes capacidades, ritmos y estilos de aprendizaje, motivaciones e intereses, situaciones sociales, étnicas, de inmigración y de salud del alumnado e incluyendo el principio de compensación.

- Decreto 68/2007, de 29 de mayo de 2007 por el que se establece y ordena el currículo de la Educación primaria de la Comunidad Autónoma de Castilla-La Mancha en el que se asegure el éxito educativo de todo el alumnado a través de la respuesta a sus necesidades específicas de apoyo educativo en un modelo inclusivo.

- Ley $7 / 2010$, de 20 de julio de Educación de Castilla-La Mancha en la que se indica que la diversidad es un elemento potenciador del enriquecimiento mutuo y que debe apoyarse en la equidad.

\section{Castilla-León}

- Decreto 40/2007, de 3 de mayo, por el que se establece el currículo de la Educación Primaria en la Comunidad de Castilla y León en el que la atención a la diversidad incluye tanto al alumnado con dificultades como al de mayor capacidad.

- Orden EDU /1152/2010, de 3 de agosto, por la que se regula la respuesta educativa al alumnado con necesidad específica de apoyo educativo escolarizado en el segundo ciclo de Educación Infantil, Educación Primaria, Educación Secundaria Obligatoria, Bachillerato y Enseñanzas de Educación Especial, en los centros docentes de la Comunidad de Castilla y León, respuesta apoyada en los principios de calidad y equidad.

\section{Extremadura}

- Decreto 82/2007, de 24 de abril, por el que se establece el Currículo de Educación Primaria para la Comunidad Autónoma de Extremadura el cual exige el respeto al derecho a la igualdad de oportunidades así como nuevas formas de adaptación e incorporación desde el respeto a la interculturalidad y a la pluralidad.

- Ley 4/2011, de 7 de marzo, de Educación de Extremadura en la que la equidad, la igualdad de oportunidades y la atención a la diversidad, relacionada con el éxito del alumno, son principios básicos de una escuela inclusiva.

La Rioja

- Decreto 4/2011, de 28 de enero, por el que se establece el Currículo de Educación Primaria en la Comunidad Autónoma de la Rioja que pone énfasis en la atención a la diversidad, la atención individualizada, la prevención y el refuerzo tempranos.

Madrid

- Orden 2316/1999, de 15 de octubre, por la que se regula el funcionamiento de las actuaciones de compensación educativa, adaptando la normativa estatal. 
- Resolución de 21 de julio de 2006, de la Viceconsejería de Educación, por la que se dictan instrucciones para la organización de las actuaciones de compensación educativa en el ámbito de la enseñanza básica en los centros docentes sostenidos con fondos públicos de la Comunidad de Madrid, actuaciones dirigidas a prevenir y compensar dificultades de acceso, permanencia y promoción en el sistema educativo.

- Decreto 22/2007, de 10 de mayo, del Consejo de Gobierno, por el que se establece para la Comunidad de Madrid el currículo de la Educación Primaria que enfatiza la atención a la diversidad, la atención individualizada, la prevención y el refuerzo tempranos.

- Orden 445/2009, de 6 de febrero, por la que se regula la incorporación tardía y la reincorporación de alumnos a la enseñanza básica del sistema educativo español estableciendo criterios uniformes.

Murcia

- Decreto 286/2007 de 7 de septiembre, por el que se establece el currículo de la Educación Primaria en la Comunidad Autónoma de la Región de Murcia que busca la atención para que todos puedan alcanzar un grado de excelencia adecuado a través de la prevención y el refuerzo.

- Decreto 359/2009, de 30 de octubre, por el que se establece y regula la respuesta educativa a la diversidad del alumnado en la Comunidad Autónoma de la Región de Murcia bajo los principios de calidad y equidad.

\section{Normativa autonómica: contenido}

En este apartado vamos a analizar el contenido de la normativa autonómica estableciendo semejanzas y diferencias, de acuerdo a los siguientes criterios:

a. Normativa autonómica posterior a la LOE sobre atención a la diversidad (en las tablas AT) y compensación educativa (en las tablas CE).

b. Unión o separación de la citada normativa autonómica.

C. Alumnado objeto de atención a la diversidad en relación con lo especificado en la LOE.

d. Alumnado objeto de compensación en relación con lo especificado en la LOE.

e. Medidas de atención a la diversidad de carácter general o específico.

f. Medidas específicas de compensación educativa.

g. Programas y/o materiales diseñados para el logro del éxito educativo de todo el alumnado 
Las tablas I a VIII muestran la descripción de los resultados obtenidos.

Tabla I. Normativa autonómica anterior/ posterior a la LOE (2006)

\begin{tabular}{|l|l|l|l|l|}
\hline Comunidad & $\begin{array}{l}\text { Ley } \\
\text { Educación }\end{array}$ & $\begin{array}{l}\text { Currículo } \\
\text { Primaria }\end{array}$ & Normativa AT & Normativa CE \\
\hline Andalucía & 2007 & 2007 & 2008 & $1999-2003$ \\
\hline Aragón & & 2007 & $2000-2010-2011$ & \\
\hline Asturias & & 2007 & & \\
\hline Canarias & & 2007 & $2007-2009-2010$ & $1995-2001-2010$ \\
\hline Cantabria & 2008 & 2007 & 2005 & 2004 \\
\hline Castilla-La Mancha & 2010 & 2007 & 2002 & $2002-2007$ \\
\hline Castilla-León & & 2007 & 2010 & 2009 \\
\hline Extremadura & 2011 & 2007 & & $2002-2007$ \\
\hline La Rioja & & 2011 & & \\
\hline Madrid & & 2007 & & $2006-2009$ \\
\hline Murcia & & 2007 & 2009 & 2009 \\
\hline
\end{tabular}

Tabla II. Normativa conjunta y/o separada

\begin{tabular}{|l|l|l|l|}
\hline Comunidad & AT + CE & AT & CE \\
\hline Andalucía & & $\mathrm{x}$ & $\mathrm{x}$ \\
\hline Aragón & & $\mathrm{x}$ & $\mathrm{x}$ \\
\hline Asturias' & $\mathrm{x}$ & & \\
\hline Canarias & $\mathrm{x}$ & & $\mathrm{x}$ \\
\hline Cantabria & $\mathrm{x}$ & & $\mathrm{x}$ \\
\hline Castilla-La Mancha & $\mathrm{x}$ & & $\mathrm{x}$ \\
\hline Castilla-León & $\mathrm{x}$ & & $\mathrm{x}$ \\
\hline Extremadura & $\mathrm{x}$ & & \\
\hline La Rioja & & & \\
\hline Madrid & & & $\mathrm{x}$ \\
\hline Murcia & $\mathrm{x}$ & & \\
\hline
\end{tabular}

${ }^{1}$ No existe normativa específica sino un conjunto de medidas de atención a la diversidad. 
Tabla III.- Alumnado incluido en la normativa de Atención a la Diversidad y conjunta (AT+CE)

\begin{tabular}{|c|c|c|c|c|c|c|c|c|c|c|c|c|}
\hline & $\begin{array}{l}\text { ơ } \\
\text { ò }\end{array}$ & 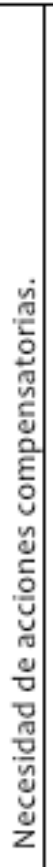 & 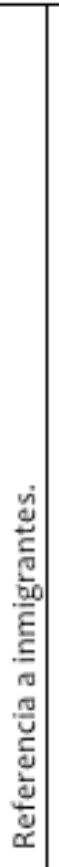 & 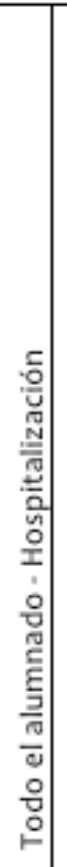 & 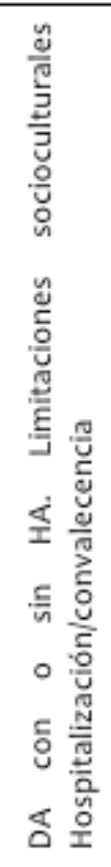 & 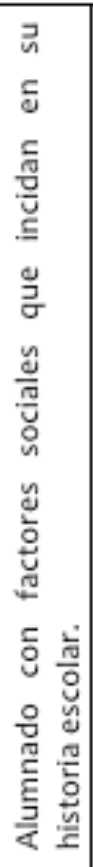 & 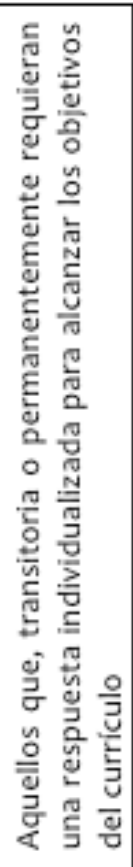 & 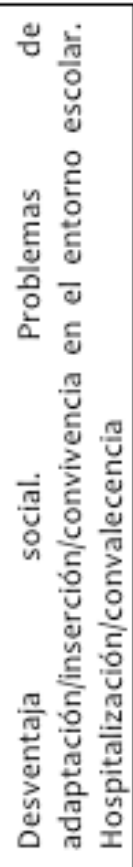 & 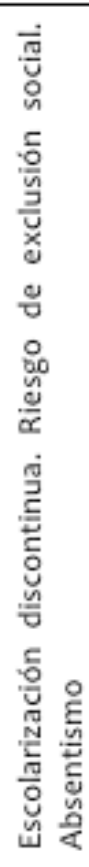 & & & 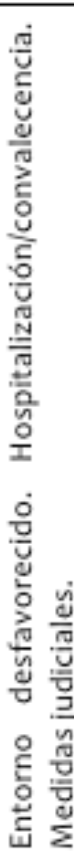 \\
\hline \multirow{5}{*}{ 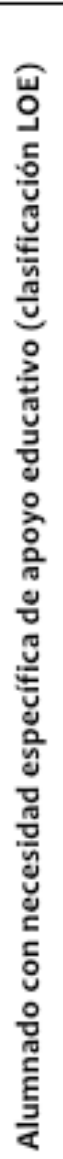 } & 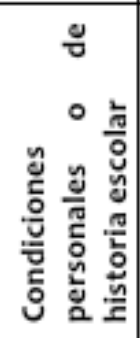 & & $\times$ & & $\times$ & $\times$ & & $\times$ & & $\times$ & $\times$ & \\
\hline & 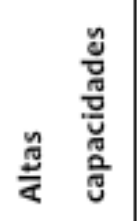 & $\times$ & $\times$ & $\times$ & 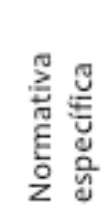 & $\times$ & $\times$ & $\times$ & $\times$ & $\times$ & $\times$ & $\times$ \\
\hline & 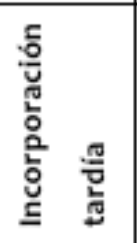 & $\times$ & $\times$ & $\times$ & $\times$ & $\times$ & & $\times$ & $\times$ & $\times$ & $\times$ & $\times$ \\
\hline & 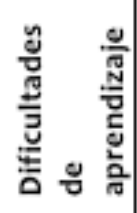 & & $\times$ & $\times$ & $\times$ & $\times$ & & $\times$ & & $\times$ & $\times$ & $\times$ \\
\hline & 㞮 & $\times$ & $\times$ & $\times$ & $\times$ & $\times$ & $\times$ & $\times$ & $\times$ & $\times$ & $\times$ & $\times$ \\
\hline & 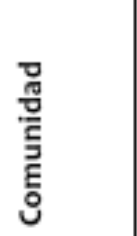 & $\begin{array}{l}\frac{\pi}{y} \\
\frac{\pi}{\pi} \\
\frac{\pi}{0} \\
\frac{c}{<}\end{array}$ & $\begin{array}{l}5 \\
0 \\
0 \\
\frac{0}{4} \\
\end{array}$ & 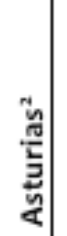 & 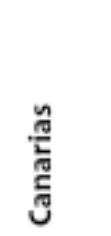 & 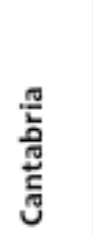 & 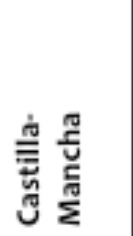 & 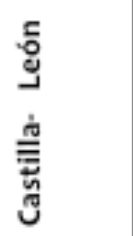 & 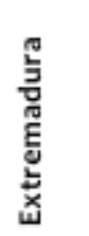 & 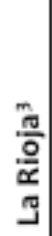 & 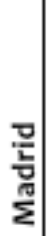 & 吾 \\
\hline
\end{tabular}


Tabla IV.- Alumnado incluido en la normativa específica de Compensación Educativa

\begin{tabular}{|c|c|c|c|c|c|c|c|c|c|c|c|c|}
\hline & $\begin{array}{l}\tilde{n} \\
0 \\
0 \\
0\end{array}$ & 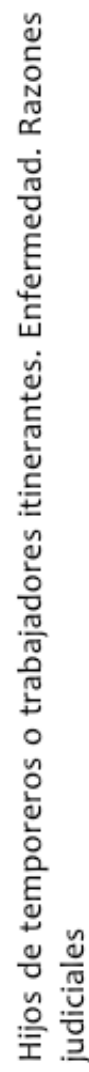 & 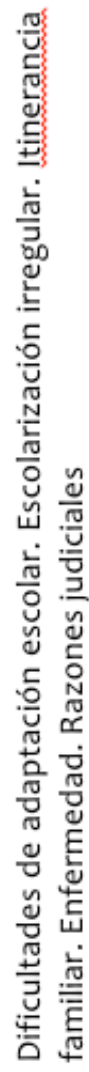 & & 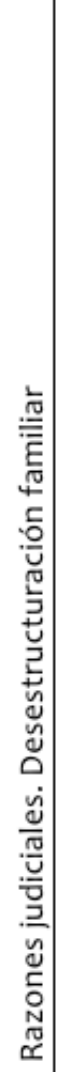 & 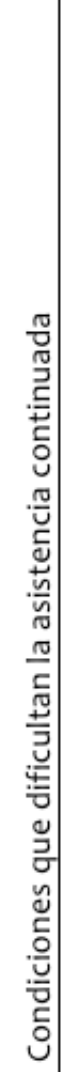 & 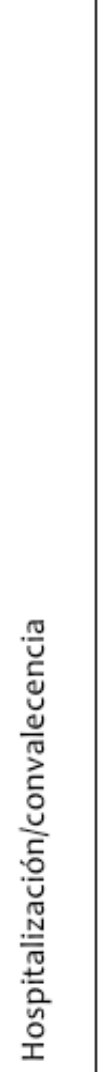 & 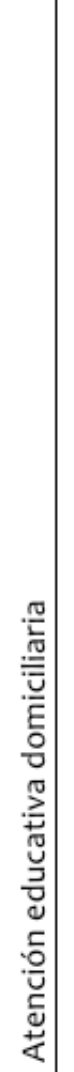 & 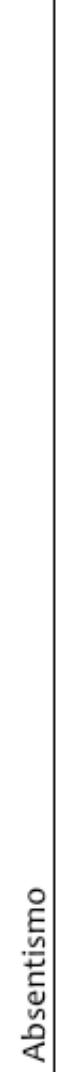 & & 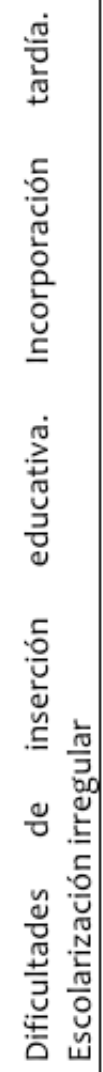 & \\
\hline$\underset{\overleftarrow{t}}{\stackrel{u}{0}}$ & 占 & $\times$ & $\times$ & & $\times$ & & & $\times$ & & & $\times$ & \\
\hline 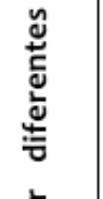 & 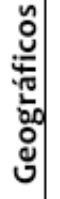 & $\times$ & & & $\times$ & & & & & & $\times$ & \\
\hline $\begin{array}{l}a \\
y \\
\frac{a}{0} \\
\frac{\pi}{\pi}\end{array}$ & 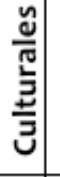 & $\times$ & $\times$ & & $\times$ & $\times$ & & $\times$ & & & $\times$ & \\
\hline $\begin{array}{l}\frac{\bar{g}}{\bar{y}} \\
\bar{v} \\
\delta \\
\delta \\
0 \\
0\end{array}$ & 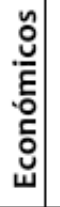 & & & & $\times$ & $\times$ & & & & & $\times$ & \\
\hline 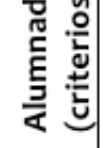 & 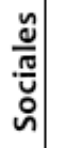 & $\times$ & $\times$ & & $\times$ & $\times$ & & $\times$ & & & $\times$ & \\
\hline & 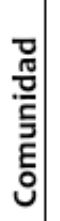 & 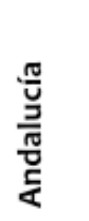 & $\begin{array}{l}\text {.0 } \\
\frac{0}{0} \\
\frac{0}{4}\end{array}$ & 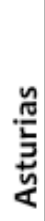 & 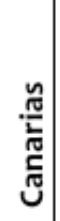 & 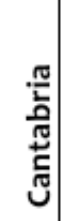 & 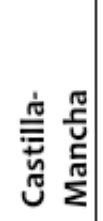 & 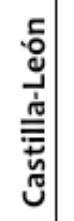 & 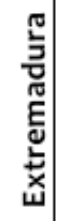 & $\begin{array}{l}\frac{\pi}{0} \\
\frac{0}{\alpha} \\
ت\end{array}$ & $\begin{array}{l}\frac{\pi}{\frac{\pi}{2}} \\
\frac{\pi}{2} \\
\sum\end{array}$ & $\frac{\pi}{5}$ \\
\hline
\end{tabular}

Tabla V.- Medidas y/o programas de atención a la diversidad generales/ordinarias 


\begin{tabular}{|c|c|c|c|c|c|c|c|c|c|c|c|}
\hline ờ & 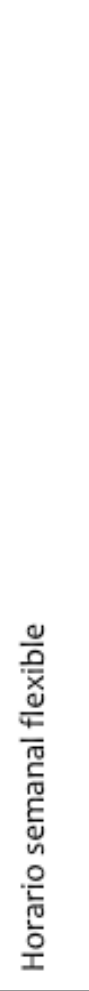 & & 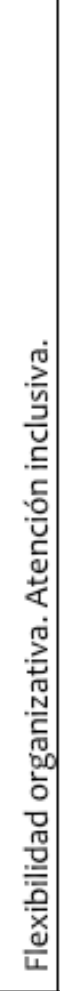 & 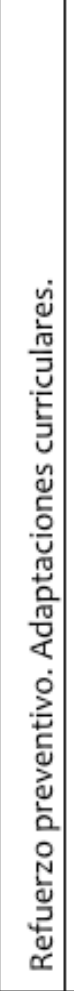 & 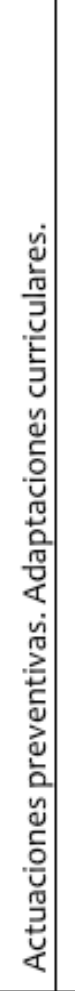 & 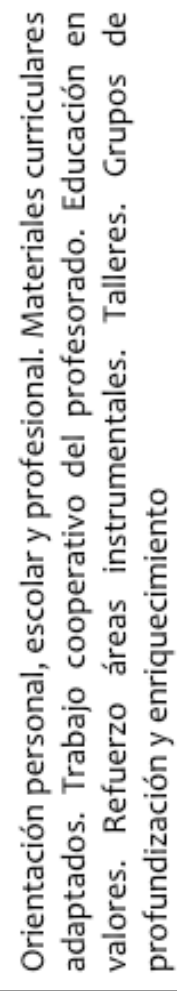 & 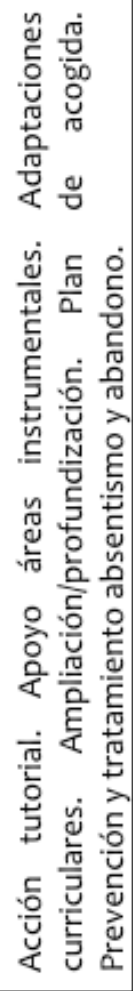 & 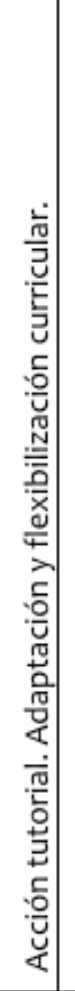 & 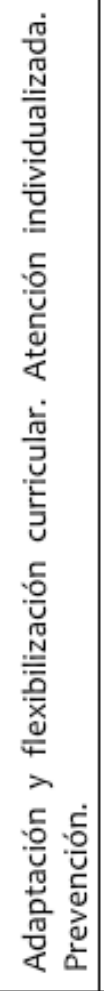 & 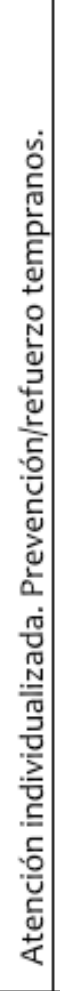 & 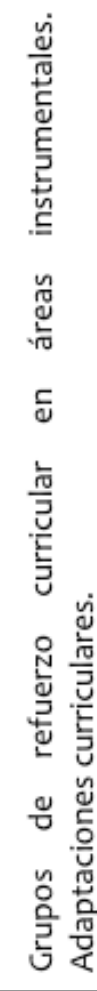 \\
\hline 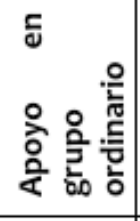 & $\times$ & & $\times$ & $\times$ & & $\times$ & $\times$ & & & $\times$ & $\times$ \\
\hline 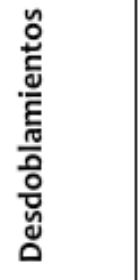 & 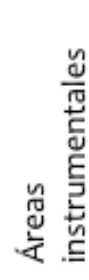 & & $\times$ & & & $x$ & & & & & $\times$ \\
\hline 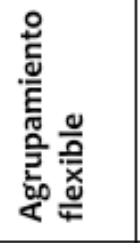 & $\times$ & & $\times$ & $\times$ & & $\times$ & $\times$ & & & $\times$ & $\times$ \\
\hline 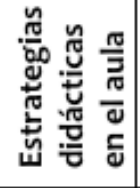 & & & & $\times$ & & $\times$ & $\times$ & & & & $\times$ \\
\hline 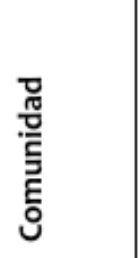 & $\frac{\frac{\pi}{y}}{\frac{3}{\pi}}$ & $\begin{array}{l}0 \\
0 \\
0 \\
0 \\
\frac{0}{\alpha}\end{array}$ & 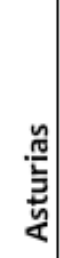 & 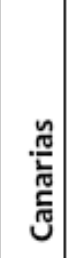 & 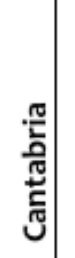 & 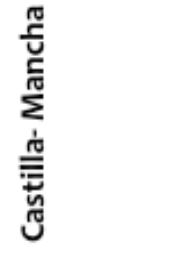 & 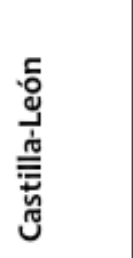 & 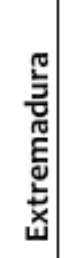 & $\begin{array}{l}\frac{\pi}{0} \\
\frac{0}{\pi} \\
\pi\end{array}$ & 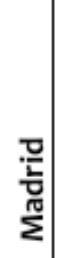 & $\stackrel{n}{\Sigma}$ \\
\hline
\end{tabular}


Tabla VI.- Medidas y/o programas de atención a la diversidad específicas/extraordinarias

\begin{tabular}{|c|c|c|c|c|c|c|c|c|c|c|c|}
\hline $\begin{array}{l}\text { Oे } \\
\text { Oे }\end{array}$ & & & 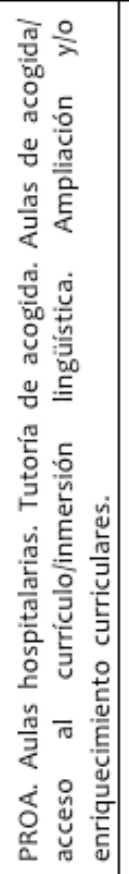 & 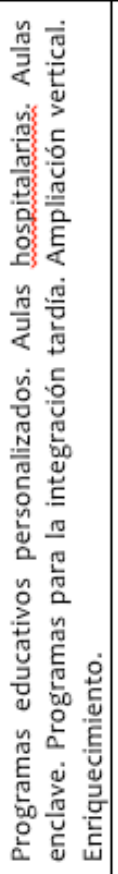 & 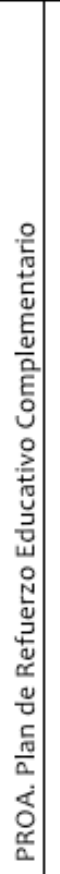 & 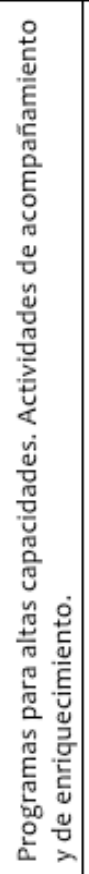 & 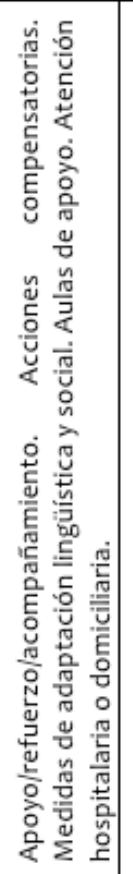 & 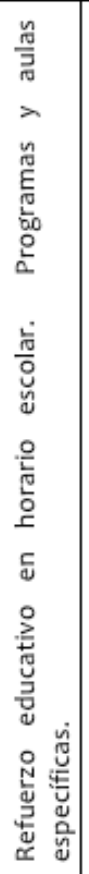 & 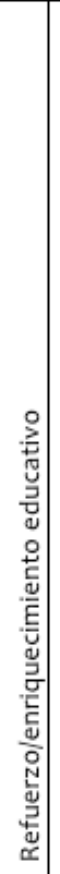 & 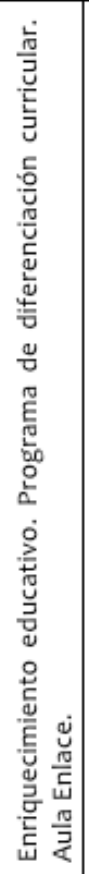 & 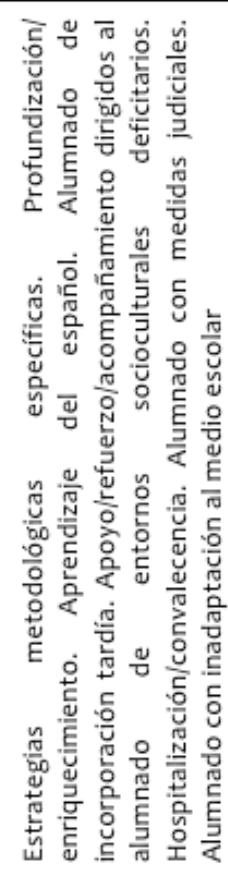 \\
\hline 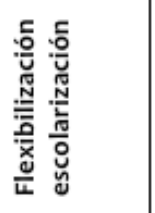 & $\times$ & $\times$ & $\times$ & $\times$ & & $\times$ & $\times$ & $\times$ & & $\times$ & \\
\hline 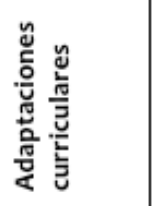 & $\times$ & $\times$ & $\times$ & $\times$ & $\times$ & $\times$ & $\times$ & $\times$ & & $\times$ & $\times$ \\
\hline 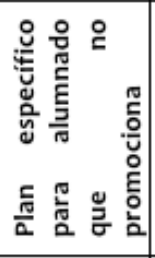 & $\times$ & & $\times$ & & $\times$ & $\times$ & & & & & \\
\hline 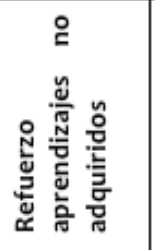 & $\times$ & & $\times$ & & $\times$ & & & & & & \\
\hline 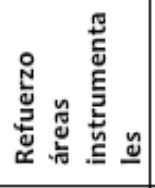 & $\times$ & & & & & & & & & & \\
\hline 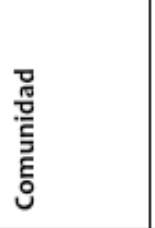 & 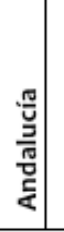 & 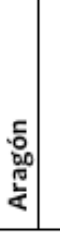 & 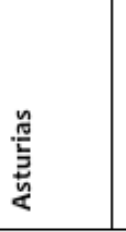 & 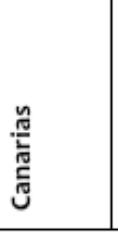 & 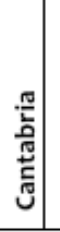 & 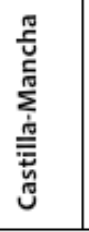 & 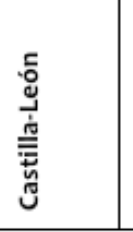 & 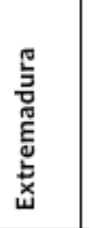 & $\begin{array}{l}\frac{\pi}{\circ} \\
\frac{a}{\alpha} \\
\Xi\end{array}$ & $\begin{array}{l}\text { D } \\
\text { L } \\
\sum \\
\end{array}$ & 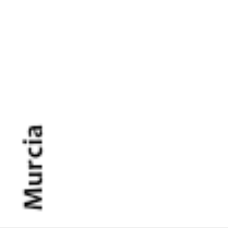 \\
\hline
\end{tabular}


Tabla VII.- Medidas específicas de compensación educativa

\begin{tabular}{|c|c|c|c|c|c|c|c|c|c|c|c|}
\hline 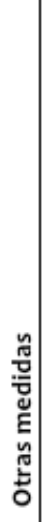 & 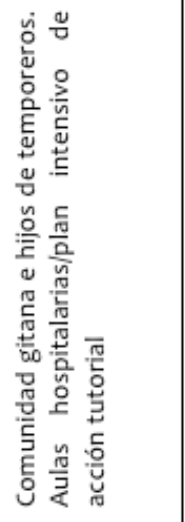 & 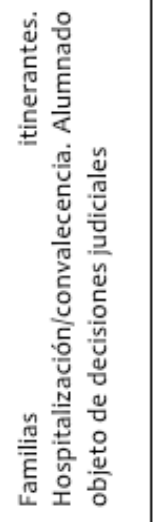 & & 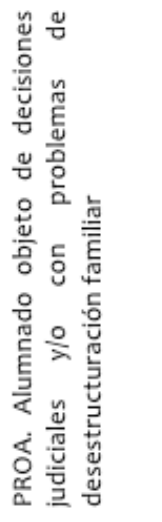 & 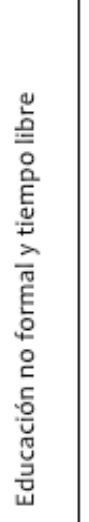 & 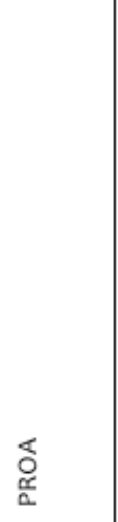 & 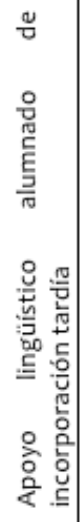 & & & 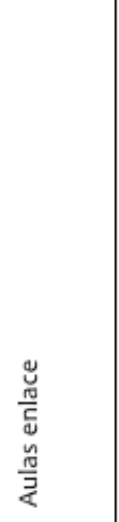 & 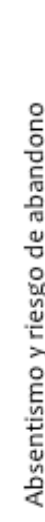 \\
\hline$\frac{\pi}{\frac{\pi}{4}}$ & 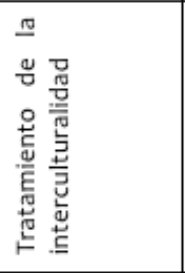 & 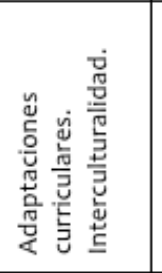 & & & & & & & & 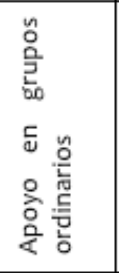 & \\
\hline 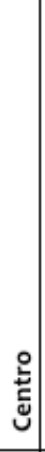 & 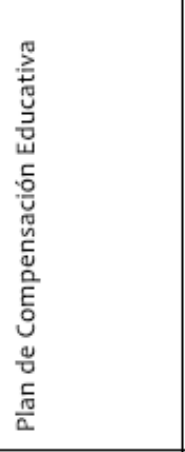 & 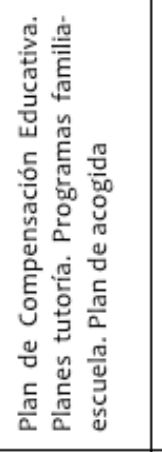 & & 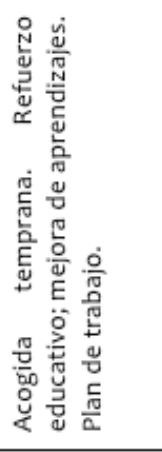 & 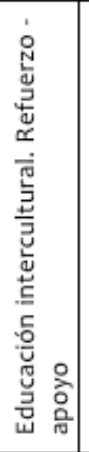 & 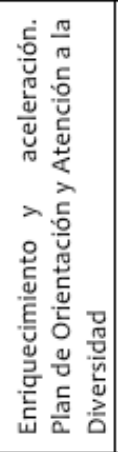 & 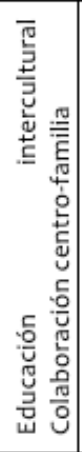 & 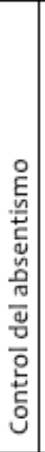 & & 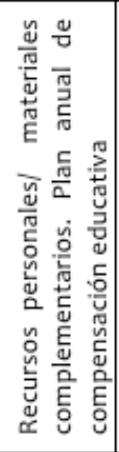 & 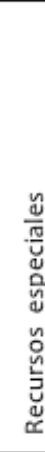 \\
\hline 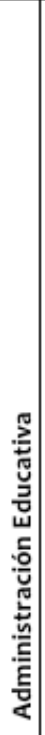 & 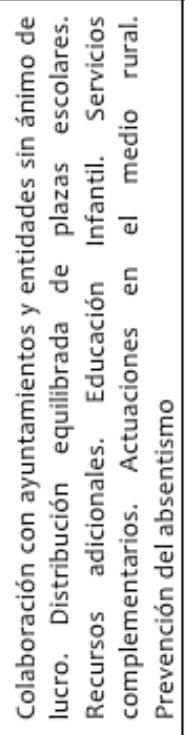 & 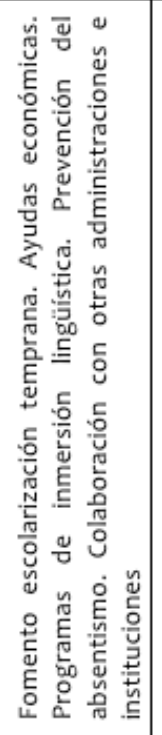 & & 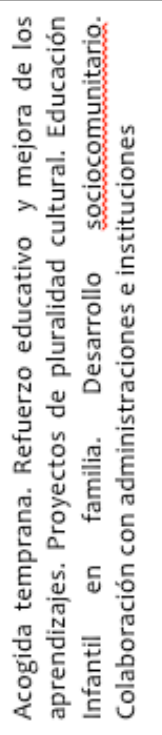 & 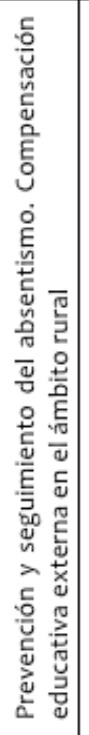 & 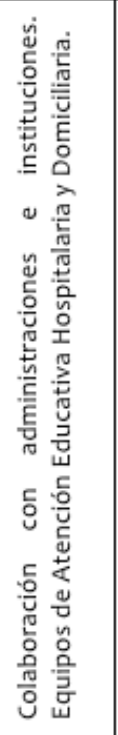 & 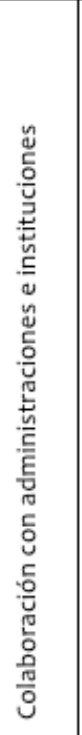 & 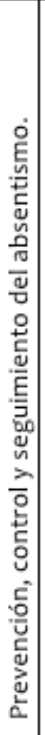 & 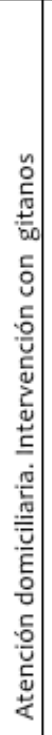 & 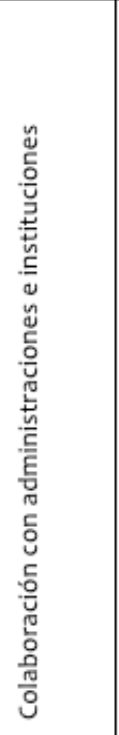 & 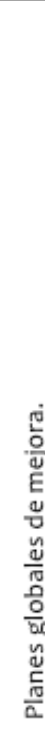 \\
\hline 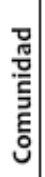 & 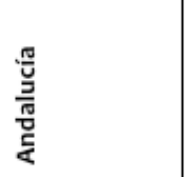 & 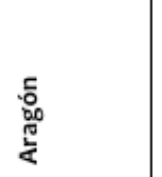 & & $\begin{array}{l}\text { 营 } \\
\text { 尊 }\end{array}$ & 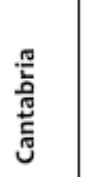 & 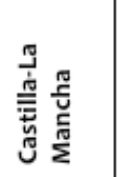 & 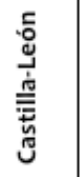 & 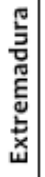 & 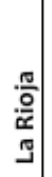 & 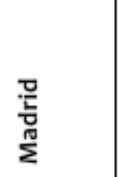 & تת \\
\hline
\end{tabular}


Tabla VIII.- Programas, estructuras organizativas y/o materiales autonómicos

\begin{tabular}{|c|c|c|c|}
\hline Comunidad & $\begin{array}{l}\text { Documentos/ } \\
\text { Materiales }\end{array}$ & Estructuras & Programas /Proyectos \\
\hline Andalucía & $\begin{array}{ll}\text { Guía } & \text { para } \\
\text { Inmigrantes. } & \text { Guía } \\
\text { Educación } & \\
\text { Intercultural } & \\
\end{array}$ & & \\
\hline Aragón & $\begin{array}{lr}\text { Programa } & \text { de } \\
\text { transición } & \text { de } \\
\text { primaria } & \text { a } \\
\text { secundaria } & \\
\end{array}$ & $\begin{array}{l}\text { Centro Aragonés de } \\
\text { Recursos para la } \\
\text { Educación Intercultural }\end{array}$ & Desarrollo de capacidades \\
\hline Asturias & $\begin{array}{llr}\text { Catálogo } & & \text { de } \\
\text { Medidas } & & \text { de } \\
\text { Atención a } & \text { la } \\
\text { Diversidad } & & \\
\end{array}$ & & \\
\hline Canarias & $\begin{array}{l}\text { Guía para la } \\
\text { detección temprana }\end{array}$ & & $\begin{array}{l}\text { Contratos-programa con centros } \\
\text { para el incremento del éxito } \\
\text { escolar } \\
\begin{array}{l}\text { Enseñanza activa de las } \\
\text { matemáticas }\end{array}\end{array}$ \\
\hline Cantabria & & & $\begin{array}{l}\text { Plan de Refuerzo Educativo } \\
\text { Complementario. Plan de } \\
\text { Interculturalidad }\end{array}$ \\
\hline $\begin{array}{l}\text { Castilla-La } \\
\text { Mancha }\end{array}$ & $\begin{array}{l}\text { Guía de apoyo a } \\
\text { Planes de Trabajo } \\
\text { Individualizado }\end{array}$ & & Aula Virtual Cervantes \\
\hline Castilla-León & & $\begin{array}{l}\text { Centro de Recursos de } \\
\text { Educación Intercultural. } \\
\text { Equipos de atención } \\
\text { especializados }\end{array}$ & Programa ALISO \\
\hline Extremadura & $\begin{array}{lr}\text { Plan Marco de } \\
\text { Atención a la } \\
\text { Diversidad }\end{array}$ & & Plan Regional sobre Absentismo \\
\hline La Rioja & & & $\begin{array}{l}\text { Proyecto de bibliotecas escolares } \\
\text { en los centros públicos. Proyecto } \\
\text { de innovación : refuerzo o } \\
\text { desarrollo de competencias en } \\
\text { horario extraescolar }\end{array}$ \\
\hline Madrid & & & $\begin{array}{l}\text { Programas de compensación } \\
\text { externa. Programa de } \\
\text { enriquecimiento del alumnado con } \\
\text { altas capacidades. Programa de } \\
\text { diferenciación curricular. Programa } \\
\text { "Para que nadie se quede atrás" }\end{array}$ \\
\hline Murcia & $\begin{array}{l}\text { Guía de Información } \\
\text { Educativa } \\
\text { Multilingüe de la } \\
\text { Región de Murcia }\end{array}$ & $\begin{array}{l}\text { Centro de animación y } \\
\text { documentación } \\
\text { intercultural. Centros } \\
\text { de recursos de } \\
\text { educación especial }\end{array}$ & Aula Virtual de Español \\
\hline
\end{tabular}




\section{Discusión y conclusiones}

- Existen pocas CCAA que tengan una normativa sobre atención a la diversidad y/o compensación educativa adaptada a la LOE. Esta situación no resulta extraña ante las continuas reformas educativas planteadas a nivel estatal y que impiden planteamientos normativos a largo plazo.

- Aquellas CCAA que tienen una normativa más antigua siguen manteniendo una mayor diferenciación entre atención a la diversidad y compensación educativa, aunque la tendencia actual es a incluir bajo el epígrafe de atención a la diversidad y/o educación inclusiva a todo el alumnado, cualquiera que sean sus características personales y/o sociales.

- La existencia de una normativa excesivamente reglamentista va en contra del principio de autonomía de los centros educativos a la hora de realizar una atención individualizada que tenga en cuenta la diversidad de intereses, necesidades y expectativas.

- Los conceptos de equidad, calidad, inclusión y éxito están estrechamente unidos a cualquier actuación en materia de atención a la diversidad y/o compensación educativa. Sin embargo su interpretación puede verse condicionada por matices que pueden tergiversar su puesta en práctica.

- Los planteamientos del centro como estructura básica de la acción educativa están en la base del principio del éxito de todo el alumnado, lo que debe conducir a un mayor conocimiento de las características que debe tener dicha estructura.

- La implicación de todo el profesorado es un requisito básico para el éxito de cualquier actuación en materia de atención a la diversidad, por lo que el trabajo cooperativo debe ser un elemento fundamental en la formación inicial y permanente de aquel.

- Las relaciones familia-escuela están en la base de la calidad de cualquier programa de apoyo y refuerzo, lo que implica profundizar en los mecanismos más adecuados para su existencia.

- La incorporación del alumnado con altas capacidades a los programas de atención a la diversidad es un hecho incuestionable.

- El alumnado con incorporación tardía al sistema educativo es objeto tanto de medidas de atención a la diversidad como de compensación educativa.

- Las medidas de compensación educativa se ajustan cuando las diferencias de origen afectan a la historia escolar del alumnado.

- Los continuos cambios legislativos, tanto a nivel estatal como autonómico, dan lugar a desfases e incongruencias en la aplicación de determinados programas. En el ámbito de la atención a la diversidad este hecho supone un retroceso importante, teniendo en cuenta la necesidad de actuaciones a largo plazo.

- La falta de sistemas de evaluación interna y externa de los programas y medidas de atención a la diversidad y/o compensación educativa es un hecho por lo que no es posible establecer mecanismos de mejora.

- La investigación educativa es necesaria en dos frentes: uno en el de la clarificación conceptual y terminológica, y otro en la evaluación de programas. 


\section{Bibliografía}

Decreto de 19 de diciembre de 2000, del Gobierno de Aragón, de atención al alumnado con necesidades educativas especiales, Boletín Oficial de Aragón, 27 diciembre 2000, 154. Disponible en: http://www.boa.aragon.es/

Decreto $138 / 2002$, de 8 de octubre por el que se ordena la respuesta educativa a la diversidad del alumnado en la Comunidad Autónoma de Castilla-La Mancha, Diario Oficial de Castilla La Mancha, 11 octubre 2002, 126. Disponible en: http://docm.jccm.es/portaldocm/

Decreto 167/2003, de 17 de junio por el que se establece la ordenación de la atención educativa a los alumnos y alumnas con necesidades educativas especiales asociadas a condiciones sociales desfavorecidas, Boletín Oficial de la Junta de Andalucía, 23 junio 2003, 118. Disponible en: http://www.juntadeandalucia.es/eboja

Decreto 98/2005, de 18 de agosto, de ordenación de la atención a la diversidad en las enseñanzas escolares y la educación preescolar en Cantabria, Boletín Oficial de Cantabria, 29 agosto 2005, 165. Disponible en: http://boc.cantabria.es/boces/

Decreto 82/2007, de 24 de abril, por el que se establece el Currículo de Educación Primaria para la Comunidad Autónoma de Extremadura, Diario Oficial de Extremadura, 3 mayo 2007, 50. Disponible en: http://doe.juntaex.es/

Decreto 40/2007, de 3 de mayo, por el que se establece el currículo de la Educación Primaria en la Comunidad de Castilla y León, Boletín Oficial de Castilla y León, 9 de julio de 2007, 130. Disponible en http://bocyl.jcyl.es/

Decreto 56/2007, de 10 de mayo, por el que se establece el currículo de la Educación Primaria en la Comunidad Autónoma de Cantabria, Boletín Oficial de Cantabria, 24 mayo 2007, 100. Disponible en http://boc.cantabria.es/boces/

Decreto 22/2007, de 10 de mayo, del Consejo de Gobierno, por el que se establece para la Comunidad de Madrid el currículo de la Educación Primaria, Boletín Oficial de la Comunidad de Madrid, 29 mayo 2007, 126. Disponible en http://www.bocm.es/bocm/Satellite?language=es\&pagename=Boletin/Page/BOCM home

Decreto 56/2007, de 24 de mayo, que regula la ordenación y establece el currículo de Educación Primaria en el Principado de Asturias, Boletín Oficial del Principado de Asturias, 16 de junio de 2007, 140. Disponible en: https://sede.asturias.es/portal/site/Asturias/menuitem.048b5a85ccf2cf40agbe6aff10 oooof7/?vgnextoid=c0C756a575acd010VgnVCM100000bb030a0aRCRD\&i18n.http.la ng=es\&calendarioPqBopa=true

Decreto 126/2007, de 24 de mayo, por el que se establece la ordenación y el currículo de la Educación Primaria, Boletín Oficial de Canarias, 6 junio 2007, 112. Disponible en http://www.gobcan.es/boc/

Decreto 68/2007, de 29 de mayo de 2007 por el que se establece y ordena el currículo de la Educación primaria de la Comunidad Autónoma de Castilla-La Mancha, Diario Oficial de Castilla La Mancha, 1 junio 2007,116. Disponible en: http://docm.jccm.es/portaldocm/

Decreto 230/2007, de 31 de julio, por el que se establece la ordenación y las enseñanzas correspondientes a la educación primaria en Andalucía, Boletín Oficial de la Junta de Andalucía, 8 agosto 2007, 156. Disponible en: http://www.juntadeandalucia.es/eboja 
Decreto 286/2007 de 7 de septiembre, por el que se establece el currículo de la Educación Primaria en la Comunidad Autónoma de la Región de Murcia, Boletín Oficial de la Región de Murcia, 12 septiembre 2007, 211. Disponible en http://www.borm.es/borm/vista/principal/inicio.jsf

Decreto 359/2009, de 30 de octubre, por el que se establece y regula la respuesta educativa a la diversidad del alumnado en la Comunidad Autónoma de la Región de Murcia, Boletín Oficial de la Región de Murcia, 3 noviembre 2009, 254. Disponible en: http://www.borm.es/borm/vista/principal/inicio.jsf

Decreto 104/2010, de 29 de julio, por el que se regula la atención a la diversidad del alumnado en el ámbito de la enseñanza no universitaria de Canarias, Boletín Oficial de Canarias, 6 agosto 2010, 154. Disponible en: http://www.gobcan.es/boc/

Decreto 4/2011, de 28 de enero, por el que se establece el Currículo de Educación Primaria en la Comunidad Autónoma de la Rioja, Boletín Oficial de La Rioja, 4 febrero 2011, 16. Disponible en: http://www.larioja.org/npRioja/default/defaultpage.jsp?idtab=449881

Dieterlen, P. (2001). Derechos, necesidades básicas y obligación institucional En A. Ziccardi (comp.), Pobreza, desigualdad social y ciudadanía. Los límites de las políticas sociales en América Latina (pp. 13-22) Buenos Aires: CLACSO.

Gordon, S. (2001). Ciudadanía y derechos sociales: ¿criterios distributivos? En A. Ziccardi (comp.), Pobreza, desigualdad social y ciudadanía. Los límites de las políticas sociales en América Latina (23-36). Buenos Aires: CLACSO.

Ley 9/1999, de 18 de noviembre, de Solidaridad en la Educación, Boletín Oficial de la Junta de Andalucía, 2 diciembre 1999, 140. Disponible en: http://www.juntadeandalucia.es/eboja

Ley Orgánica 2/2006, de 3 de mayo de Educación, Boletín Oficial del Estado, 4 mayo 2006, 106. Disponible en: http://www.boe.es/

Ley 17/2007, de 10 de diciembre, de Educación de Andalucía, Boletín Oficial de la Junta de Andalucía, 26 diciembre 2007, 252. Disponible en: http://www.juntadeandalucia.es/eboja

Ley 6/2008, de 26 de diciembre, de Educación de Cantabria, Boletín Oficial de Cantabria, 30 diciembre 2008, 251. Disponible en: http://boc.cantabria.es/boces/

Ley $7 /$ 2010, de 20 de julio de Educación de Castilla-La Mancha, Diario Oficial de Castilla La Mancha, 28 junio 2010, 144. Disponible en: http://docm.jccm.es/portaldocm/

Ley 4/2011, de 7 de marzo, de Educación de Extremadura, Diario Oficial de Extremadura, 9 marzo 2011, 47. Disponible en: http://doe.juntaex.es/

López Melero, M. (1997). La educación intercultural: el valor de la diferencia. En J. A. Torres, M. Román y E. Rueda (Eds), La innovación de la Educación Especial (pp. 173-199). Jaén: Universidad de Jaén.

LLorent García V. \& López Azuaga, R. (2012). Demandas de la formación del profesorado. El desarrollo de la educación inclusiva en la Educación Secundaria Obligatoria. REIFOP 15(3), 27-34. Recuperado de http://www.aufop.com.

Orden de 19 de mayo de 1995, por la que se establecen las acciones de carácter específico de compensación educativa en la Comunidad Autónoma de Canarias, Boletín Oficial de Canarias, 21 junio 1995, 77. Disponible en : http://www.gobcan.es/boc/

Orden 2316/1999, de la Consejería de Educación, de 15 de octubre, por la que se regula el funcionamiento de las actuaciones de compensación educativa, Boletín Oficial de la Comunidad de Madrid, 25 octubre 1999, 253. Disponible en: 
http://www.bocm.es/bocm/Satellite?language=es\&pagename=Boletin/Page/BOCM home

Orden de 17 de junio de 2004, por la que se establecen las bases y se convoca a los Centros Educativos Públicos de Cantabria para la realización de actuaciones de Compensación Educativa, Boletín Oficial de Cantabria, 28 junio 2004. Disponible en: http://boc.cantabria.es/boces/

Orden de 9 de mayo de 2007, del Departamento de Educación Cultura y Deporte, por la que se aprueba el currículo de la educación primaria, Boletín Oficial de Aragón, 1 junio 2007, 65. Disponible en: http://www.boa.aragon.es/

Orden de 25 de julio de 2008 , por la que se regula la atención a la diversidad que cursa la educación básica en los centros docentes públicos de Andalucía, Boletín Oficial de la Junta de Andalucía, 22 agosto de 2008, 167. Disponible en: http://www.juntadeandalucia.es/eboja

Orden 445/2009, de 6 de febrero, por la que se regula la incorporación tardía y la reincorporación de alumnos a la enseñanza básica del sistema educativo español, Boletín Oficial de la Comunidad de Madrid, 19 febrero 2009, 42. Disponible en: http://www.bocm.es/bocm/Satellite?language=es\&pagename=Boletin/Page/BOCM home

Orden EDU/34/2009, de 6 de abril, por la que se regula el Plan de Refuerzo Educativo Complementario en el Sistema Educativo de Cantabria, Boletín Oficial de Cantabria, 15 abril 2009, 171. Disponible en:http://boc.cantabria.es/boces/

Orden de 7 de junio de 2010, por la que se regulan las condiciones que han de regir los planes, programas y proyectos de intervención dirigidos a mejorar la calidad y equidad en educación, de aplicación en los centros docentes no universitarios sostenidos con fondos públicos de la Comunidad Autónoma de Canarias, Boletín Oficial de Canarias, 15 junio 2010, 116. Disponible en: http://www.gobcan.es/boc/

Orden EDU/1152/2010, de 3 de agosto, por la que se regula la respuesta educativa al alumnado con necesidad específica de apoyo educativo escolarizado en el segundo ciclo de Educación Infantil, Educación Primaria, Educación Secundaria Obligatoria, Bachillerato y Enseñanzas de Educación Especial, en los centros docentes de la Comunidad de Castilla y León, Boletín Oficial de Castilla y León, 13 agosto 2010, 156. Disponible en: http://bocyl.jcyl.es/

Orden de 13 de diciembre de 2010, por la que se regula la atención al alumnado con necesidades específicas de apoyo educativo en la Comunidad Autónoma de Canarias, Boletín Oficial de Canarias, 22 diciembre 2010, 250. Disponible en: http://www.gobcan.es/boc/

Organización de las Naciones Unidas para la Educación, la Ciencia y la Cultura (UNESCO) (1994). Declaración y Marco de Acción sobre Educación para todos. Conferencia Mundial. Dakar, Senegal, Septiembre.

Resolución de 21 de julio de 2006, de la Viceconsejería de Educación por la que se dictan instrucciones para la organización de las actuaciones de compensación educativa en el ámbito de la enseñanza básica en los centros docentes sostenidos con fondos públicos de la Comunidad de Madrid, Boletín Oficial de la Comunidad de Madrid, 2 noviembre 2006, 192. Disponible en: http://www.bocm.es/bocm/Satellite?language=es\&pagename=Boletin/Page/BOCM home

Resolución de 9 de febrero de 2011, de la Dirección General de Ordenación, Innovación y Promoción educativa, por la que se dictan instrucciones sobre los procedimientos y 
los plazos para la atención educativa al alumnado con necesidades específicas de apoyo educativo en los centros escolares de la Comunidad Autónoma de Canarias, Boletín Oficial de Canarias, 24 febrero 2011, 40. Disponible en http://www.gobcan.es/boc/ 


\section{Autores}

Dra Pilar Abós Olivares

Profesora Titular de Universidad en la Facultad de Ciencias Sociales y Humanas de Teruel, perteneciente a la Universidad de Zaragoza. Directora del Máster Propio en Educación y Desarrollo Rural. Docencia en el Grado de Magisterio de Educación Primaria y en el Máster de Profesorado de Educación Secundaria. Vinculada a los procesos de Gestión de la Calidad de la Universidad de Zaragoza. Investigación vinculada a la escuela rural y a las metodologías didácticas relacionadas con el desarrollo de competencias

Dra Virginia Domingo Cebrián

Profesora Asociada en la Facultad de Ciencias Sociales y Humanas de Teruel, perteneciente a la Universidad de Zaragoza. Docencia en el Grado de Magisterio de Educación Infantil. Investigación vinculada a la escuela rural y a la etapa de educación infantil 